\title{
Glucose-dependent insulinotropic polypeptide concentration is influenced by the proportion of palmitic acid in the $s n-2$ position of dietary TAGs
}

\author{
A. Filippou ${ }^{1}$, S. E. Berry ${ }^{1}$, S. Baumgartner ${ }^{2}$, R. P. Mensink ${ }^{2}$ and T. A. B. Sanders ${ }^{1}$ \\ ${ }^{1}$ Diabetes and Nutritional Sciences Division, King's College London, Franklin-Wilkins Building, London SE1 9NH and \\ ${ }^{2}$ Nutrition and Toxicology Institute Maastricht, University of Maastricht, Netherlands
}

Plant and animal sources of palmitic acid differ in TAG structure and this may influence their respective effects on postprandial metabolism $^{(1)}$. Palmitic acid is found predominantly in the $s n-2$ position in animal fats, but predominantly in the $s n-1$ and $s n-3$ positions in plant fats. Dietary TAG is a potent stimulator of glucose-dependent insulinotropic polypeptide (GIP) in human subjects ${ }^{(2)}$; however, the effects of TAG structure on its release are uncertain. Postprandial changes in plasma TAG ( $8 \mathrm{~h})$ and GIP (3h) concentrations were assessed following four meals containing $50 \mathrm{~g}$ test fat provided as high oleic sunflower oil (HOS) (control), palm olein (PO), randomly interesterified palm olein (IPO) and lard using a randomised crossover design in healthy men $(n 25)$ and women ( $n$ 25). The lard contained a similar proportion of SFA $(46.0 v .44 .8 \mathrm{wt} \%)$ and oleic acid $(40.0 v .42 .6 \mathrm{wt} \%)$ to PO and IPO. The proportion of palmitic acid in the $s n-2$ position was $9.4 \%, 39.6 \%$ and $71.5 \%$ in the PO, IPO and lard, respectively.
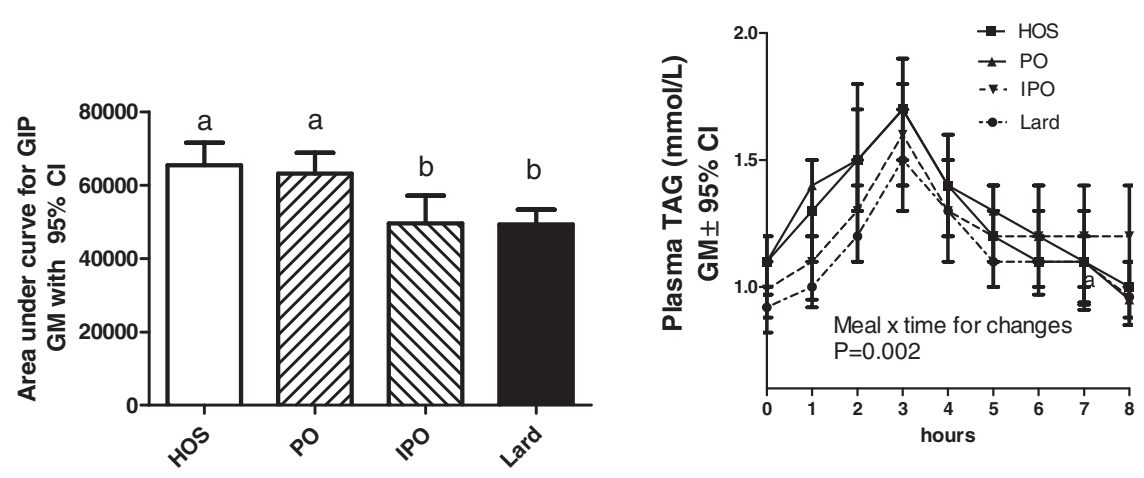

Bars with different letters are significantly different from each other $P<0.01$; Bonferroni comparison test.

GIP secretion was decreased by fats with a high content of palmitic acid in the $s n-2$ position (lard and IPO) compared to control and PO. The percentage differences $(95 \% \mathrm{CI})$ for the integrated area under the curve compared with the control were: lard $-28.4 \%$ $(-41.9,-14.5 ; P<0.001)$; IPO $-27.4(-41.3,-14.3 ; P<0.001)$. Both IPO and lard showed a less rapid rise in plasma TAG compared with the control and PO. The integrated area under the curve for TAG $(95 \% \mathrm{CI})$ following lard differed by $-14.2 \%(-24.3,-4.1) v$. PO and by $-11.2 \%(-21.7,-0.9) v$. control. These findings indicate that TAG rich in palmitic acid in the $s n-2$ position inhibit GIP release and that GIP release is associated with an early rise in plasma TAG. Furthermore, the physical structure of TAG may modulate the release of gut peptides and thus influence postprandial metabolism.

This work was supported by the Malaysian Palm Oil Board.

1. Berry SE, Woodward R, Yeoh C, Miller GJ \& Sanders TA (2007) Lipids 42, 315-323.

2. Drucker DJ (2007) J Clin Invest 117, 24-3. 